\title{
The Association between Lifestyle Factors and the Risk of Developing Breast Cancer in Jordanian Women
}

\author{
Reema I. Mahmoud ${ }^{1)}$, Lina S. Marei ${ }^{2)}$, Muna H. Shareef ${ }^{3)}$, Reema F. Tayyem ${ }^{1)}$ \\ ${ }^{1)}$ Department of Nutrition and food technology, Faculty of agriculture, The University of Jordan \\ ${ }^{2)}$ King Hussein Cancer Center, Amman, Jordan \\ ${ }^{3)}$ Jordan Ministry of Health: at AL-Bashir Hospital
}

\begin{abstract}
Background and Aims: Breast cancer (BC) is the most common type of cancer that affects Jordanian women. Several studies highlighted the independent effect of various diet and lifestyle factors on the risk of BC. Therefore, the objective of this study was to evaluate the association between lifestyle factors and BC in a selected group of Jordanian woman.

Methods: A case control study was conducted from October 2016 to September 2017. A total of 400 Jordanian women 20-65 years of age were recruited. 200 of recently diagnosed BC patients were compared to 200 apparently healthy controls. BC patients were matched with controls in age, income and marital status. A package consisted of three structured questionnaires: Personal Information and Seven-Day Physical Activity Recall (PAR) questionnaires were administered to all participants in this study.

Results: Increasing in BMI was associated significantly and positively with BC risk (OR 1.80 (95\%CI: 1.01-3.27), P-trend $=0.037)$. A significant inverse association has been found between BC and physical activity (OR 0.32 (95\%CI: $0.13-0.80)$, P-trend $=0.021$ ). A significant positive association was detected between BC and smoking (OR 4.38 (95\%CI: 2.08-9.22), $\mathrm{P}$-trend $=0.001)$, a significant negative effect of lactation in the risk of BC was found (OR 3.71(95\%CI: 2.20-6.26), P-trend $=0.0001$.

Conclusion: A significant increase in BC risk was detected with Increasing in BMI and smoking, on the other hand a significant negative effect of lactation and physical activity in the risk of $\mathrm{BC}$ was found.
\end{abstract}

Keywords: Breast Cancer, Lifestyle, BMI, Smoking, Physical activity

(Received January 27, 2020; Accepted February 13, 2020)

\section{Introduction}

Breast cancer (BC) is the most frequently diagnosed cancer and the leading cause of cancer deaths in women worldwide $^{1)}, \mathrm{BC}$ is the most common type of cancer that affects Jordanian women and one of the top five cancers in Jordan ${ }^{2}$. About 14.1 million new cancer cases and 8.2 million deaths occurred in 2012 worldwide ${ }^{1)}$. Breast cancer $(\mathrm{BC})$ is the most common type of cancer that affects all women as well as Jordanian women and one of the top five cancers in Jordan ${ }^{2}$. In the year 2014 there, were 1187 newly diagnosed cases of BC in both sexes accounting for $20.8 \%$ of all newly diagnosed cancer cases ${ }^{2}$.

Many lifestyle factors are likely to enhance the risk of developing $\mathrm{BC}^{3)}$. Evidence reported by many researchers who found that risk of $\mathrm{BC}$ was elevated in smokers and strong evidence suggested a relationship between ciga-

Corresponding Author: Reema Tayyem, Professor of Nutrition, Department of Nutrition and Food Technology, Faculty of Agriculture, The University of Jordan, Amman, Jordan. Email: r.tayyem@ju.edu.jo rette smoking and increased $\mathrm{BC}$ risk $^{4-6)}$. The association of cigarette smoking with $\mathrm{BC}$ risk has been long suggested because carcinogens found in tobacco smoke may pass into the blood stream and be transported to the breast ${ }^{5)}$. Components of cigarette smoke have been detected in fluid from breast ducts of smokers ${ }^{4}$. There is strong scientific evidence that not having children or having them after the age of 30 years, avoiding breast feeding, prolonged use of the contraceptive pills, and having hormone replacement therapy (HRT) after menopause all increase the incidence of $\mathrm{BC}^{3,7,8)}$. Zhou et al. ${ }^{9)}$ revealed that ever breastfeeding had a reduced risk of $\mathrm{BC}$ compared with never breastfeeding. The inverse association was also found for the longest compared with shortest categories of breastfeeding ${ }^{9)}$. For the physical activity (PA), Ma et al showed that substantially increasing the amount of total recreational $\mathrm{PA}$ in adulthood decreases the risk of $\mathrm{BC}^{10)}$. A recent meta-analysis pointed out that PA was significantly associated with reduced the risk of BC in their meta-analysis and they advised that PA should be advocated for the primary prevention of $\mathrm{BC}^{11)}$. Their evidence showed that a linear relationship was found between 
breast cancer risk and PA (recreational activity and total activity), and the risk was reduced by $3 \%$ for every 10 metabolic equivalent of energy hours per week increment in recreational and total $\mathrm{PA}^{11)}$. Therefore, the objectives of this study were to evaluate the association between the levels of PA, mass index (BMI); and some lifestyle factors and the risk of $\mathrm{BC}$ among Jordanian women.

\section{Materials and methods}

\section{Study Design}

A case-control design was used to determine the dietary and lifestyle risk factors associated with BC risk in a conveniently selected sample from Jordanian women.

\section{Sample}

Two hundred patients who were recently diagnosed (up to 3 months from diagnosis) with $\mathrm{BC}$ were included conveniently in the study; they were taken from King Hussein Cancer Center (KHCC) and Al-Bashir Hospital, the control group was included from the community (various health centers were directing by the center for early detection of $\mathrm{BC}$, the employees and visitors in KHCC and Al-Bashir Hospital, as well as patients' accompanying persons). The researcher asked the participants in control group if they had been undergone a prior clinical examination or mammography during the last year before participating in the study. The ratio was one for the cases to one for the control group (1:1). However, matching between the patients and control subjects was done on the following variables; age, income categories, marital status. The inclusion criteria for the patients: recently diagnosed with $\mathrm{BC}$ up to 3 months from diagnosis, Jordanian nationality, aged between 20 and 65 years and able to communicate verbally. Exclusion criteria are: critically or terminally ill, hospitalized, unable to communicate verbally, suffering from other types of cancer and other diseases require specific diet, patients who decided to incomplete the interview or refused to participate in the study, patients who given unrealistic answers during the interview and recently diagnosed patients who were older than 65 years.

\section{Setting}

For the cases, the hospital setting was utilized for data collection. Hospitals that include oncology units were included (specialized oncology hospitals KHCC and Al-Bashir Hospital). The outpatient department in each hospital was the setting for data collection. Permission from each hospital for having a private room with good physical condition in order to carry out the interviews was obtained.

For the controls, various health centers and the outpatient department in each hospital (KHCC and Al-Bashir Hospital) were the setting for data.

\section{Data Collection}

A structured questionnaire was used to collect the data from patient and controls; Personal Information Sheet and Seven-Day Physical Activity Recall (PAR) ${ }^{12)}$. An interview technique was used as method for data collection and the questionnaire was filed by the researcher.

\section{Personal Information Sheet}

This sheet was composed of questions related to age, marital status, education, employment, family income/ month, residency area and house condition, smoking status, medication and previous and current health problems. Additionally questions related to parity \& gravidity, lactation, use of contraceptives and gynecologic history were added for married women.

\section{Physical Activity Levels}

The Physical Activity Recall (PAR), developed by Sallis et al. for the Stanford Five-City Project, has been used with adult populations for several projects ${ }^{12)}$. When using PAR, it is necessary to consider the frequency, intensity, time, and type of the physical activity. The PAR interview focuses on collecting data on intensity, time or duration, and type of activity. The 7-day PAR is a structured interview that depends on participant's recall of time spent engaging in PA over a seven day period. It covers different levels of PA such as aerobic exercise, work-related activities, gardening, walking, recreation, and leisure-time activities ${ }^{12}$. We measured PA using a resting metabolic score (MET), one MET is equivalent to quiet sitting, which for the average adult is approximately equals to $4.184 \mathrm{~kJ} \bullet \mathrm{kg}-1$ body weight $\bullet \mathrm{h}-1$ or $3.5 \mathrm{ml}$ of oxygen $\bullet \mathrm{kg}$ body weight-1 $\bullet$ min- 1 . The number of hours spent in sleep and different activity levels are obtained and converted into Kilocalories. Time spent in sleeping (1 MET), light (1.5 METs), moderate (4 METs), hard (6 METs), and very hard (10 METs) activities for the past 7 days are multiplied by their respective MET values and then summed. An estimate of total kilocalories of energy expenditure per day was calculated.

\section{Anthropometric measurements}

Measurement of weight was taken to the nearest 0.1 $\mathrm{kg}$, with minimal clothing and without shoes, using a calibrated portable scale and height was measured to the nearest $0.5 \mathrm{~cm}$ with participants in the full standing position without shoes using a calibrated portable measuring rod, both were conducted in order to define BMI (calculated as the ratio of weight in kilograms to the square of height in meters), the measurement for each participant was taken in duplicate by the researcher.

\section{Human Protection}

The representatives from each hospital were calling each participant in order to invite them for participat- 
ing in the research. During the first meeting with the participants, the investigator was explained the purpose of the study and allowing them to read the consent form before signing it. The participant understood that she can withdraw from the study at any time she want and this was not harm them in any way. The potential risks and potential benefits also were explained in details to know that the present study has no risk. A signed consent was obtained before the data collection. Obtained patients' information was treated confidentially. However, only the investigator knew patients' names and she was the only one who gave them ID. All used tools and instruments (questionnaires) were labeled with patient number (ID).

\section{Study Procedure}

Each oncologist participating in the study was contacted through his hospital (KHCC and Al-Bashir Hospital) for getting a permission to collect data at the outpatient department and to facilitate having a private room for the interviews. The proposal was presented and approved to the IRB of each hospital.

\section{Statistical analysis}

Descriptive analyses were conducted to examine the frequency of different variables. Chi-square was used to detect the differences among categorical variables. T-test was used to find the difference between continuous variables of cases and controls which are presented as Mean \pm SD. Logistic regression was used to calculate odds ratio $\mathrm{OR}, \mathrm{CI}$ and $\mathrm{p}$-value for trend with age (continuous), BMI (continuous), physical activity level (continuous), total energy intake (continuous), occupation, education level, marital status and family history for the BC participants were evaluated as potential confounders. The significance level was set at $\mathrm{p}<0.05$. All statistical analyses were conducted in SPSS version 22.0 (IBM SPSS Statistics for Windows, IBM Corporation).

\section{Results}

The results of this case-control study highlighted the association between some dietary and lifestyle risk factors for developing BC. Table 1 Shows participants' age, anthropometric measurements, socio-demographic and health characteristics. Average age for cases was $48.9 \pm$ 0.63 years and $47.5 \pm 0.59$ years for control. Significant difference was found between cases and controls in BMI. No significant differences were detected in marital status and monthly income. However, smoking, education level, employment status, family history of $\mathrm{BC}$, contraceptive use, lactation and hormone replacement therapy were significantly different as $\mathrm{BC}$ cases compared to the controls. Table 2 illustrates the ORs and corresponding 95\% CI of the $\mathrm{BC}$ and control group for deferent lifestyle factors (after adjusting for potential confounders) moving from normal body weight to overweight or obesity in BMI categories were significantly associated with BC (OR 1.80 (95\%CI: 1.01-3.27), P-trend $=0.037)$. A significant inverse association has been found between $\mathrm{BC}$ and PA (OR 0.32 (95\% CI: 0.13-0.80), P-trend $=0.021$ ). In addition, a significant association was detected between $\mathrm{BC}$ and smoking (OR 4.38 (95\%CI: 2.08-9.22), P-trend $=0.001$ ). Moreover, a significant protective effect of duration of lactation in months from 1-6, from 7-12 and from 19-36 months were found (OR 0.29 (95\%CI: 0.09-0.87), OR 0.39 (95\%CI: 0.18-0.84) and OR 0.51 (95\%CI: 0.28-0.93) respectively, P-trend $=0.0001$ ). According to number of pregnancies, the study results showed that one to three pregnancies could be significantly protect form BC (OR 0.27 (95\%CI: 0.12-0.62), P-trend $=0.001$ ). However, more than seven pregnancies were found to increase the risk of BC (OR 1.79 (95\%CI: 0.77-4.13), P-trend $=0.001)$. Using contraceptive of hormonal tablets type (OR 1.1 (95\%CI: 0.38-3.13)) or hormonal lobe (OR 2.07 (95\%CI: 0.16-27.47)) were not significantly associated with risk of BC. However, the trend of using contraceptives may significantly increase the risk of BC especially for hormonal types.

\section{Discussion}

The results of this study accumulate the evidence for the presence of an association between BC risk with diet and lifestyle behaviors. Generally, the findings of this case-control study on BC risk highlight the relationship between lifestyle factors and this type of cancer among Jordanian females.

In this study, we found a significant difference between cases and controls in BMI, in addition to that, the risk of $\mathrm{BC}$ increased as the BMI moves from normal body weight to overweight or obesity. Many studies reported that $\mathrm{BMI}$ was associated with the risk of $\mathrm{BC}^{3,13,14)}$. A plausible explanation for the association between BMI and body weight on BC risk could be as suggested by several studies; that obesity may contribute in increasing the risk of $\mathrm{BC}$ by development of localized inflammation $^{13)}$, increasing in local estrogen production, changes in cellular metabolism ${ }^{12}$, increasing in insulin and other growth factor secretion ${ }^{13)}$ and increasing in leptin production $^{14)}$. Our study revealed an inverse association between $\mathrm{PA}$ and BC. This is in agreement with several studies which indicated that PA was significantly associated with reduced risk of $\mathrm{BC}^{16-19)}$. PA might reduce the $\mathrm{BC}$ risk through several biological mechanisms, including the impact of PA on adiposity ${ }^{19)}$, sex hormones ${ }^{17)}$, insulin resistance ${ }^{18)}$, adipokines, and inflammatory markers ${ }^{16}$.

When contrasting about the relation between $\mathrm{BC}$ and lifestyle factors, our study found a significant direct association between $\mathrm{BC}$ and smoking, which means that current and former smokers from BC group was higher 
Table 1. Characteristics of the study participants

\begin{tabular}{|c|c|c|c|}
\hline \multirow{2}{*}{ Variables } & \multicolumn{2}{|c|}{ Treatments } & \multirow{2}{*}{ p-value } \\
\hline & Cases $(n=200)$ & Controls $(n=200)$ & \\
\hline \multicolumn{4}{|l|}{ Mean \pm SEM } \\
\hline Age (y) & $48.9 \pm 0.63$ & $47.5 \pm 0.59$ & 0.106 \\
\hline Height $(\mathrm{cm})$ & $159.3 \pm 0.42$ & $161.7 \pm 0.41$ & 0.001 \\
\hline Weight $(\mathrm{Kg})$ & $75.7 \pm 1.05$ & $73.2 \pm 0.95$ & 0.080 \\
\hline $\mathrm{BMI}\left(\mathrm{kg} / \mathrm{m}^{2}\right)$ & $29.8 \pm 0.39$ & $27.9 \pm 0.36$ & 0.001 \\
\hline Number of pregnancy & $4.6 \pm 0.23$ & $3.4 \pm 0.20$ & 0.001 \\
\hline Number of miss carriage & $1.1 \pm 0.11$ & $0.90 \pm 0.09$ & 0.226 \\
\hline Duration of lactation (months) & $8.2 \pm 0.54$ & $7.7 \pm 0.55$ & 0.599 \\
\hline Physical activity (METs) & $1425.38 \pm 133.34$ & $3464.72 \pm 1205.57$ & 0.019 \\
\hline \multicolumn{4}{|l|}{$\mathbf{N}(\%)$} \\
\hline \multicolumn{4}{|l|}{ Marital Status } \\
\hline Married & $154(77.0)$ & $156(78.0)$ & \multirow{4}{*}{0.789} \\
\hline Single & $26(13.0)$ & $29(14.5)$ & \\
\hline Divorce & $8(4.0)$ & $5(2.50)$ & \\
\hline Widow & $12(6.0)$ & $10(5.0)$ & \\
\hline \multicolumn{4}{|l|}{ Education Level } \\
\hline Illiterate & $12(6.0)$ & $5(2.5)$ & \multirow{7}{*}{0.001} \\
\hline School & $52(26.0)$ & $12(6.0)$ & \\
\hline High school & $65(32.5)$ & $46(23.0)$ & \\
\hline Diploma & $47(23.5)$ & $65(32.5)$ & \\
\hline Bachelor & $14(7.0)$ & $55(27.5)$ & \\
\hline Master degree & $7(3.5)$ & $12(6.0)$ & \\
\hline Doctorate degree & $3(1.5)$ & $5(2.5)$ & \\
\hline \multicolumn{4}{|l|}{ Work Status } \\
\hline Yes & $53(26.5)$ & $89(44.5)$ & \multirow{2}{*}{0.001} \\
\hline No & $147(73.5)$ & $111(55.5)$ & \\
\hline \multicolumn{4}{|l|}{ BMI categories } \\
\hline Under weight & $0(0.0)$ & $1(0.5)$ & \multirow{4}{*}{0.013} \\
\hline Normal weight & $33(16.5)$ & $58(29.0)$ & \\
\hline Overweight & $91(45.5)$ & $70(35.0)$ & \\
\hline Obese & $76(38.0)$ & $71(35.0)$ & \\
\hline \multicolumn{4}{|l|}{ Smoking } \\
\hline Yes & $40(20.0)$ & $14(7.0)$ & \multirow{2}{*}{0.001} \\
\hline No & $160(80.0)$ & $186(93.0)$ & \\
\hline \multicolumn{4}{|c|}{ Family members diagnosed with cancer } \\
\hline Yes & $109(54.5)$ & $73(36.5)$ & 0.001 \\
\hline No & $91(45.5)$ & $127(63.5)$ & \\
\hline Type of cancer that your family $n$ & vith & & \\
\hline Not having cancer & $88(44.0)$ & $126(36.0)$ & \\
\hline Gastric & $2(1.0)$ & $3(1.5)$ & \\
\hline Colon & $9(4.5)$ & $13(6.5)$ & \\
\hline Pancreatic & $2(1.0)$ & $4(2.0)$ & 0.001 \\
\hline Breast & $65(32.5)$ & $31(15.5)$ & \\
\hline Others & $34(17.0)$ & $23(11.5)$ & \\
\hline Contraceptive use & & & \\
\hline Yes & $103(52.0)$ & $83(41.5)$ & 0.001 \\
\hline No & $97(48.0)$ & $117(58.5)$ & \\
\hline Type of contraceptive & & & \\
\hline Not used & $97(48.0)$ & $117(52.0)$ & \\
\hline Lobe & $50(25.0)$ & $52(26.0)$ & \\
\hline Hormonal lobe & $2(1.0)$ & $1(0.5)$ & 0059 \\
\hline Tablet & $37(18.7)$ & $20(10.0)$ & 0.059 \\
\hline Injection & $1(0.5)$ & $0(0.0)$ & \\
\hline Others & $13(6.5)$ & $10(5.0)$ & \\
\hline Lactation & & & \\
\hline Not Having child & $4(2.0)$ & $45(22.5)$ & \\
\hline Yes & $130(65.0)$ & $131(65.5)$ & 0.001 \\
\hline No & $66(33.0)$ & $24(12.0)$ & \\
\hline Hormonal replacement therapy u & & & \\
\hline Use & $48(24.0)$ & $23(11.1)$ & 0.001 \\
\hline Not use & $152(76.0)$ & $177(88.9)$ & 0.001 \\
\hline
\end{tabular}

Significance is at $\mathrm{p} \leq 0.05$ 
Table 2. The Association between Lifestyle Factors and BC risk

\begin{tabular}{|c|c|c|c|c|}
\hline Factor & $\begin{array}{c}\text { Cases } \\
(\mathrm{n}=\mathbf{2 0 0})\end{array}$ & $\begin{array}{l}\text { Controls } \\
(\mathrm{n}=\mathbf{2 0 0})\end{array}$ & $* \mathrm{OR}(95 \% \mathrm{CI})$ & p-trend \\
\hline \multicolumn{5}{|l|}{ Body Mass Index } \\
\hline Normal weight & 33 & 58 & 1 & \multirow{3}{*}{0.037} \\
\hline Overweight & 91 & 70 & $1.80(1.01-3.27)$ & \\
\hline Obese & 76 & 71 & $1.51(0.82-2.78)$ & \\
\hline \multicolumn{5}{|l|}{ Physical Activity } \\
\hline Inactive & 181 & 163 & 1 & \multirow{3}{*}{0.021} \\
\hline Minimally active & 8 & 24 & $0.32(0.13-0.80)$ & \\
\hline Health Enhancing physical activity & 9 & 13 & $0.41(0.15-1.13)$ & \\
\hline \multicolumn{5}{|l|}{ Smoking } \\
\hline No & 160 & 186 & 1 & \multirow{2}{*}{0.001} \\
\hline Yes & 40 & 14 & $4.38(2.08-9.22)$ & \\
\hline \multicolumn{5}{|l|}{ Number of pregnancies } \\
\hline 0 & 35 & 44 & 1 & \multirow{5}{*}{0.001} \\
\hline $1-3$ & 34 & 70 & $0.27(0.12-0.62)$ & \\
\hline $4-6$ & 77 & 59 & $1(0.48-2.11)$ & \\
\hline $7-10$ & 46 & 24 & $1.79(0.77-4.13)$ & \\
\hline $10<$ & 8 & 3 & $2.12(0.45-9.88)$ & \\
\hline \multicolumn{5}{|l|}{ Type of contraceptive } \\
\hline Not used & 97 & 117 & 1 & \multirow{5}{*}{0.001} \\
\hline Lobe & 50 & 52 & $0.7(0.27-1.85)$ & \\
\hline Hormonal lobe & 2 & 1 & $2.07(0.16-27.47)$ & \\
\hline Pills & 37 & 20 & $1.1(0.38-3.13)$ & \\
\hline Others & 13 & 10 & $0.69(0.27-1.81)$ & \\
\hline \multicolumn{5}{|l|}{ Duration of lactation(months) } \\
\hline 0 & 70 & 69 & 1 & \multirow{5}{*}{0.001} \\
\hline $1-6$ & 26 & 37 & $0.29(0.09-0.87)$ & \\
\hline $7-12$ & 61 & 59 & $0.39(0.18-0.84)$ & \\
\hline $13-18$ & 33 & 16 & $0.44(0.18-1.06)$ & \\
\hline $19-36$ & 10 & 19 & $0.51(0.28-0.93)$ & \\
\hline
\end{tabular}

* OR is adjusted for age, martial statues, education level, work status, monthly income, physical activity, smoking, family history, health problem, number of pregnancy, lactation, contraceptives, hormonal replacement therapy.

Significance is at $\mathrm{p} \leq 0.05$

when compared with controls, and the risk of $\mathrm{BC}$ increased $(\mathrm{OR} 4.38$, P-trend $=0.001)$. The same result was reported by many researchers who suggested a relationship between cigarette smoking and increased BC risk $^{4,5,6,20)}$. The main mechanisms which have explained the association of cigarette smoking with $\mathrm{BC}$ risk, have been suggested by Nyante et $a l^{4)}$ and Dossus et $a l^{5)}$ as carcinogens found in tobacco smoke may pass into the blood stream and be transported to the breast also components of cigarette smoke have been detected in fluid from breast ducts of smokers ${ }^{4,5)}$. Also the finding of this study highlights the presence of significant differences in HRT, contraceptive use and family history between BC cases and controls. Well-known risk factors of $\mathrm{BC}$ have been identified, including $\mathrm{HRT}^{3)}$, contraceptive use ${ }^{7}$, and family history of $\mathrm{BC}^{8)}$. One of the main findings of this study is that cases and controls showed significant reduction in $\mathrm{BC}$ risk in all duration of lactation. This finding is in agreement with the previous finding by Zhou et al. ${ }^{9)}$ who indicated that ever breastfeeding had a reduced risk of $\mathrm{BC}$ compared with never breastfeeding. The inverse association was also found for the longest compared with shortest categories of breastfeeding ${ }^{9}$. Several mecha- nisms have been proposed to explain the observed association by reduced exposure to the cyclic hormones of reproductive life due to ovulatory suppression ${ }^{9)}$, and an expression of transforming growth factors in human BC cells, during breastfeeding ${ }^{3)}$. In another study, Butt et $a l$. ${ }^{7}$ found a controversial result from this study and concluded that there was no association between duration of lactation and $\mathrm{BC}$ risk $^{7)}$.

In a study of this type we rely greatly on the ability and memory recall of participants to accurately and carefully provide information from a period when it was not necessarily important to remember the details of long physical activities that were undertaken. It is understandable that some individuals may have a greater recall than others, and that biases may exist in the minds of those being interviewed, and indeed, by the interviewer. Also, we attempted to control for a range of potential confounders. We did not measure alcohol use (culturally discouraged). There were just two women who consume alcohol. We did not take into account the possible effects of BC stage, type or the treatment on dietary and life style patterns. The selection bias was high.

Confirmative studies should verify and extend the pre- 
sented data on Jordanian dietary habits in order to establish recommendations for people in Jordan to decrease $\mathrm{BC}$ incidence.

\section{Conclusions}

The present study provides evidence supporting the presence of an association between lifestyle factors and the risk of BC. Higher BMI and physical inactivity are implicated in developing BC. Also the finding of this study documented significant associations among smoking, breast feeding, HRT, contraceptive use and family history and $\mathrm{BC}$ risk.

\section{Competing interests:}

The authors declare that they have no competing interest.

\section{Author Contributions:}

RFT and RIM participated in conception and design of the study. RIM, MHS and LSM recruited participants. RIM and MHS collected data from hospitals. RFT, RIM and LSM interpreted the results and drafted the manuscript.

Funding:

This research was funded by Deanship of Academic Research of The University of Jordan (Grant number 2016-2017/14).

\section{Acknowledgement:}

The authors would like to thank Deanship of Academic Research of The University of Jordan for supporting this project.

\section{Ethics Policies:}

The study was conducted in accordance with the ethical standards of the responsible committee on human experimentation and with the Helsinki declaration of 1975 , as revised in The proposal was approved by the Institutional Review Board of the 2 hospitals: King Hussein Cancer Center (KHCC) and Al-Basheer (16 KHCC 57 and 2250 , respectively).

\section{References}

1) Albuquerque R., Baltar V. and Marchioni D. Breast Cancer and dietary patterns: a systematic review. Nutrition Reviews, 2013; 72(1): $1-17$.

2) Cancer incidence in Jordan 2014. Ministry of Health, NonCommunicable Disease Directorate.

3) Ferrini K., Ghelfi F., Mannucci R. and Titta L. Lifestyle, nutrition and breast cancer: facts and presumptions for consideration. E-cancer, 2015; 9: 557.

4) Nyante S., Gierach G., Dallal C., Freedman N., Park Y., Danforth K., Hollenbeck A. and Brinton L. Cigarette smoking and postmenopausal breast cancer risk in a prospective cohort. British Journal of Cancer, 2014; 110, 2339-2347.

5) Dossus L., Boutron-Ruault M., Kaaks R., Gram I., Vilier A., Fervers B., Manjer J., Tjonnel A., et al. Active and passive cigarette smoking and breast cancer risk: Results from the EPIC cohort. The International Journal of Cancer, 2014; 134, 1871-1888.
6) Li B., Wang L., Lu M., Mo X., Lin F., Ho S. and Zhang C. Passive Smoking and Breast Cancer Risk among Non-Smoking Women: A Case Control Study in China. PLOS ONE, 2015; 10. 1371.

7) Butt S., Borgquist S., Anagnstaki L., Landberg G. and Manjer J. Breastfeeding in relation to risk of different Breast Cancer characteristics. BioMed Center, 2014; 7(216), 500-1756.

8) McKenzie F., Ferrari P., Freisling H., Chajès V., Rinaldi S., de Batlle J., Dahm CC., Overvad K., Baglietto L., Dartois L., Dossus L., Lagiou P., et al. Healthy lifestyle and risk of breast cancer among postmenopausal women in the European Prospective Investigation into Cancer and Nutrition cohort study. International Journal of Cancer, 2015; 136: 2640-2648.

9) Zhou Y., Chen J., Li Q., Huang W., Lan H. and Jiang H. Association between Breastfeeding and Breast Cancer Risk: Evidence from a Meta-analysis. Breast Feeding Medicine, 2015; 10(3), 175-82.

10) Ma H., Xu X., Ursin G., Simon MS., Marchbanks PA., Malone KE., Lu Y., McDonald JA., Folger SG., Weiss LK., Sullivan-Halley J., Deapen DM., Press MF., Bernstein L. Reduced risk of breast cancer associated with recreational physical activity varies by HER2 status. Cancer Med. 2015; 4(7): 1122-35.

11) Chen X., Wang Q., Zhang Y., Xie Q., Tan X. Physical Activity and Risk of Breast Cancer: A Meta-Analysis of 38 Cohort Studies in 45 Study Reports. Value Health. 2019; 22(1): 104-128.

12) Sallis J., Haskell W. and Wood P. Physical activity assessment methodology in the Five-City Project. American Journal of Epidemiology, 1985;121, 91-106.

13) Bandera E., Chandran U., Hong C., Troester M., Bethea T., AdamsCampbe L., Haiman L., Park S., Olshan A., Ambrosone C., Palmer J. and Rosenberg L. Obesity, body fat distribution, and Risk of Breast Cancer Subtypes in African American Women participating in the Amber consortium. Breast Cancer Research and Treatment, 2015; 150(3), 655-666.

14) White W., Nichols H., Bradshaw P. and Sandler D. Overall and Central Adiposity and Breast Cancer Risk in the Sister Study. Cancer Journal, 2015; 121(20), 3700-3708.

15) Boonyaratanakornkit V. and Pateetin P. The Role of Ovarian Sex Steroids in Metabolic Homeostasis, Obesity, and Postmenopausal Breast Cancer: Molecular Mechanisms and Therapeutic Implications. BioMed Research International, 2015; 1-15.

16) Zhang Y. and Kang S. Physical activity and risk of breast cancer: a meta-analysis of prospective studies. Breast cancer Research and Treatment, 2013;137: 869-882.

17) Thomson C., McCullough M., Wertheim B., Chlebowski C., Martinez M., Stefanick M., Rohan T., Manson J. and Tindle H. Nutrition and Physical Activity Cancer Prevention Guidelines, Cancer Risk, and Mortality in the Women's Health Initiative. Cancer Prevention Research, 2014; 7(1), 42.

18) Gonçalves A., Florencio G., Silva M., Cobucci R., Giraldo P. and Cote N. Effects of physical activity on breast cancer prevention: a systematic review. Journal of Physical Activity \& Health, 2014; 11(2), 445-54.

19) Kyu H., Bachman V., Alexande L., Mumford J., Afshin A., Estep K., Veerman L., Delwiche K. and Iannarone M. Physical activity and risk of breast cancer, colon cancer, diabetes, ischemic heart disease, and ischemic stroke events: systematic review and dose-response meta-analysis for the Global Burden of Disease Study 2013, British Medical Journal, 2016; 10(3857).

20) Land S., Liu Q., Wickerham L., Costantino J. and Ganz P. Cigarette smoking, physical activity, and alcohol consumption as predictors of cancer incidence among women at high risk of breast cancer in the NSABP P-1 Trial. Cancer Epidemiology, Biomarkers \& Prevention, 2014; 23(5), 823-832. 